\title{
TI.138.1
}

\section{Trust and Identity in Research and Education (TIER) Final Report to Investors}

- PDF: TIER Final Report.pdf

- Text: TIER Final Report.txt

More Information

\begin{tabular}{|l|l|}
\hline Repository ID & TI.138.1 \\
\hline Persistent URL & http://doi.org/10.26869/TI.138.1 \\
\hline Title & Trust and Identity in Research and Education (TIER) Final Report to Investors \\
\hline Authors & Bill Kaufman, Kevin Morooney, Erin Murtha, Ann West, Dean Woodbeck \\
\hline Sponsor & TIER Community Investor Council \\
\hline Review & \\
\hline Status & Preserve \\
\hline Publish Date & August 20,2019 \\
\hline DOI & $10.26869 /$ TI.138.1 \\
\hline Signature & \\
\hline Deprecated & No \\
\hline Future Review & \\
\hline Supersedes & \\
\hline Format & PDF, text \\
\hline Related Docs & \\
\hline Development Location & TIER Final Report.docx \\
\hline IP Framework & CC BY 4.0 \\
\hline Subject Tags & TIER \\
\hline Notes & \\
\hline
\end{tabular}

